Management international

International Management

Gestiòn Internacional

Stratégies de réduction des biais de la décision collaborative

à distance, vers une auto-régulation émotionnelle. Revue, clarification de la littérature et extension

Bias Reduction Strategy of Collaborative Remote Decision Towards

an Emotional Self-Regulation. Review, Clarification and Extension

Estrategia de reducción de los sesgos de la decisión colaborativa a distancia hacia una autorregulación emocional. Revista, aclaración de la literatura y extensión

Delphine van Hoorebeke

Volume 23, numéro hors-série, 2019

URI : https://id.erudit.org/iderudit/1068532ar

DOI : https://doi.org/10.7202/1068532ar

Aller au sommaire du numéro

Éditeur(s)

HEC Montréal

Université Paris Dauphine

ISSN

1206-1697 (imprimé)

1918-9222 (numérique)

Découvrir la revue

Citer cet article

van Hoorebeke, D. (2019). Stratégies de réduction des biais de la décision collaborative à distance, vers une auto-régulation émotionnelle. Revue, clarification de la littérature et extension. Management international /

International Management / Gestiòn Internacional, 23, 12-26.

https://doi.org/10.7202/1068532ar

\section{Résumé de l'article}

A l'heure des nouvelles technologies, les décisions sont devenues collaboratives à distance. Eléments clés de la décision, les stratégies de réduction de biais cognitifs aident à la gestion des phénomènes qui perturbent la prise de décision optimale. Or, ces stratégies sont peu étudiées dans la prise de décision collaborative à distance. Ainsi, une clarification des écrits sur le sujet révèle une dimension universelle et spécifique à ce type de décisions : l'émotion. Elle permet la construction d'une stratégie de réduction des biais avec ses outils managériaux pour un consensus de groupe à distance allégé en biais au travers d’une auto-régulation émotionnelle. 


\title{
Stratégies de réduction des biais de la décision collabo- rative à distance, vers une auto-régulation émotionnelle. Revue, clarification de la littérature et extension
}

\author{
Bias Reduction Strategy of Collaborative Remote Decision Towards \\ an Emotional Self-Regulation. Review, Clarification and Extension \\ Estrategia de reducción de los sesgos de la decisión colaborativa \\ a distancia hacia una autorregulación emocional. Revista, \\ aclaración de la literatura y extensión
}

\author{
DELPHINE VAN HOOREBEKE \\ Université de Toulon \\ Laboratoires Cergam France / Cirano Montréal
}

\begin{abstract}
RÉSUMÉ
A l'heure des nouvelles technologies, les décisions sont devenues collaboratives à distance. Eléments clés de la décision, les stratégies de réduction de biais cognitifs aident à la gestion des phénomènes qui perturbent la prise de décision optimale. Or, ces stratégies sont peu étudiées dans la prise de décision collaborative à distance. Ainsi, une clarification des écrits sur le sujet révèle une dimension universelle et spécifique à ce type de décisions : l'émotion. Elle permet la construction d'une stratégie de réduction des biais avec ses outils managériaux pour un consensus de groupe à distance allégé en biais au travers d'une auto-régulation émotionnelle.
\end{abstract}

Mots-Clés : Décision collaborative à distance, réduction des biais, travail émotionnel de groupe, consensus

\section{ABSTRACT}

In the age of new technologies, decisions have become not only collective, but also collaborative and computer-mediated. Key to decision-making, debiasing strategies help in the management of the phenomena which disrupt optimal decision-making. So, clarification of the literature on this subject reveals a universal and specific dimension in computer-mediated collaborative decisions: emotion. Emotion allows for the construction of a strategy of reduction of the biases of this type of decision with its managerial tools, to reach of mediated bias-free group consensus through emotional auto-regulation.

Keywords: computer-mediated collaborative decision, debiasing, emotional group labor, consensus

\section{RESUMEN}

A la hora de las nuevas tecnologías, las decisiones no solamente solo colectivas, sino colaborativas a distancia. Elementos clave de la decisión, las estrategias de reducción de sesgos cognitivos ayudan a la gestión de los fenómenos que perturban una toma de decisiones óptima. Estas estrategias son poco estudiadas en la toma de decisiones colaborativas a distancia. Así, una aclaración de lo escrito sobre el tema revela una dimensión universal y específica de este tipo de decisiones: la emoción. Ésta permite la construcción de una estrategia de reducción de los sesgos de este tipo de decisión con sus instrumentos de gestión para un consenso de grupo a distancia disminuido sesgadamente a través de una autorregulación emocional.

Palabras Clave: decisión colaborativa a distancia, limitación de los sesgos, trabajo emocional de grupo, consenso es problèmes managériaux actuels, surtout quand ils ont une
dimension internationale, sont devenus trop complexes pour
qu'une seule personne puisse les appréhender dans leur globalité
Dans de nombreux cas, une décision n'est plus portée par un
seul décideur, mais est issue d'un compromis entre plusieurs
points de vue et intérêts possiblement contraires. Aux vues des
évolutions managériales qui les accompagnent, notamment les
databases ${ }^{1}$, decision support systems ${ }^{2}$, où la décision est déléguée
à des algorithmes limitant l'incertitude, les «opendecide » favo-
risent les décisions collaboratives à distance qui font d'autan
plus souvent appel à l'expertise de plusieurs membres souvent
diversifiés. A l'instar du succès de Wikipédia à rassembler des
connaissances collaboratives, ces évolutions permettraient-elles
1. Bases de données et d'informations pour faciliter la prise de décisions
2. Système d'aide à la décision par ordinateur
3. Terme utilisé sur certains sites internet: décision ouverte au collectif d'assurer la prise de décision consensuelle, combinant ensemble les meilleures idées et les préoccupations majeures de chacun? Selon l'acception de synergie, les décisions prises en groupe auraient, en effet, tendance à être plus productives. A cet égard, Larrick (2004) considère la décision collective, comme un outil de réduction des biais individuels. Le groupe permet, selon lui, la correction d'erreurs par la complémentarité des expertises formulées. Or, cela suppose une décision consensuelle et trouver un consensus limité en biais cognitifs reste un problème non résolu et problématique pour les entreprises. Morel (2002) explique «surle plan strictement cognitif, raisonner en groupe pose des problèmes, par exemple si l'on travaille sur deux variables simultanément. Le meilleur exemple, c'est quand on essaie de rédiger un document 
à plusieurs : c'est beaucoup plus difficile, et on obtient souvent un plus mauvais résultat.». Les dirigeants de Google (Schmidt et Rosenberg, 2014) ajoutent que la plupart des managers prônent le consensus sans savoir de quoi il s'agit. Les auteurs insistent sur le sujet en s'appuyant sur l'étymologie latine du terme consensus : cum, ensemble et entire, ressentir ou penser. Cette décomposition étymologique leur permet de pointer que le terme n'indique nullement la recherche d'unanimité. A cet égard, le consensus recherché est vu comme un processus cognitif collectif (ressentir ou penser), indépendant d'un comportement moutonnier comme l'envisagent Pontikes et Barnett (2017).

Ce processus cognitif est, à présent, assimilé grâce aux descriptifs réalisés par les recherches antérieures sur la décision de groupe. Parmi elles, les études sur la décision collaborative à distance montrent que celle-ci bénéficie d'attributs spécifiques favorables. Ainsi, la perception de son efficacité est liée à une transmission sélective d'informations entre membres du groupe. Elle permet également la désinhibition lorsque qu'il y a anonymat (Jessup et al., 1990, Connolly et al., 1990). Ces études pointent également des éléments défavorables. Elle est soumise à des biais cognitifs spécifiques, tels que la déshumanisation qu'elle engendre (Dubrovsky 1985; Comtet, 2006). Néanmoins, la littérature à ces sujets peut paraitre quelque peu dépassée étant donnée l'extrême rapidité d'évolution des technologies utilisées. Ces études ont été réalisées à une époque où les outils de communication à distance étaient moins développés qu'aujourd'hui (visioconférence, chat, par exemple). Ainsi, sans nier ces études fondamentales, pour compléter la littérature existante et en réaliser l'analyse, la décision collaborative à distance est, ici, vue comme une décision de groupe pourvue de spécificités, correspondant à une agrégation de décisions individuelles diverses devant aboutir à un consensus. Imaginons une entreprise dont trois départements, marketing, $\mathrm{RH}$ et production, doivent décider d'une stratégie en commun. Ces trois départements, aux compétences diverses et de surcroit implantés dans des pays différents, vont-ils prendre une décision consensuelle, limitée en biais, pour suivre l'objectif commun? Dans un contexte de distance, il est, en effet, montré que le consensus est plus long à obtenir (Walther, 1996) et doit être supporté par des facteurs de contingence, tels l'animation par un leader. A cet effet, l'objectif, ici, est de décomposer la prise de décision collaborative à distance par la description de tous les biais auxquels elle peut être confrontée par leur possible agrégation - subordination aux biais individuels additionnés de biais collectifs et de biais spécifiques à son aspect médié par ordinateur-. L'objectif est de modéliser un processus heuristique -opération mentale- évolutif guidant la décision collaborative consensuelle et satisfaisante (Simon, 1945) et de répondre à la question : comment limiter les biais de décideurs en collaboratif à distance? La première étape pour étayer la construction d'une stratégie de réduction de biais consiste, ainsi, à détailler les trois catégories de décisions -individuelles, collectives en face à face et collaboratives à distance ${ }^{4}$, pour en repérer les effets distinctifs. Pour cela, leur définition puis une revue des études comparatives permettent d'établir la définition de la décision collaborative à distance et de montrer ses distinctions. Grâce à ces éléments, puis à une revue de leurs biais distinctifs, un point d'ancrage cognitif commun, est repéré, l'aspect émotionnel inhérent à la décision.
Cette revue ajoutée à celle de Larrick (2004) sur les outils de réduction de biais existants permet, le développement d'une stratégie de réduction des biais de la décision collaborative à distance, afin d'atteindre un consensus limité en biais grâce à un mécanisme d'auto-régulation émotionnelle.

\section{Théorie et définitions}

S'appuyant sur un raisonnement usuel «un ensemble d'individus est plus que la somme des individus qui la composent", il pourrait paraître impensable de partir de l'individuel pour atteindre l'analyse agrégée. D'un point de vue théorique, un type de modèles de la décision de groupe appuie cette vision d'une analyse agrégée, les «modèles orientés-individus» (Grimm et Railsback, 2005). Si certains modèles n'envisagent que l'aspect collectif dans leurs analyses, les modèles multi agents et plus spécifiquement, orientés-individus, aiguisent notre intérêt parce qu'ils focalisent leur étude sur le concept de «décision». Ils considèrent la décision de groupe comme l'agrégation des décisions individuelles et sont construits à partir de théories dont l'analyse se situe au niveau individuel. Les définitions de chaque type de décision étayent la nécessité de considérer d'une façon spécifique l'individu dans la décision collaborative à distance.

- la prise de décision individuelle. Roy et Bouyssou (1993) estiment que la décision est souvent présentée comme le fait d'un individu isolé (décideur) qui exerce librement un choix entre plusieurs possibilités d'actions à un moment donné dans le temps.

- la décision collective puis collaborative et à distance.

- Pour Novak et Ulfarino (2017, p.67), «Elle est entendue comme 1) un choix fait collectivement par l'ensemble des membres d'un groupe délimité, et 2) qui s'impose à ce groupe voire à la totalité des membres d'un ensemble social plus large que ce groupe représente».

- La prise de décision collaborative est envisagée par Marakas (1999) comme une activité conduite par une entité collective composée de deux ou plusieurs individus et caractérisée à la fois en termes de propriétés de l'entité collective et de celles de ses membres individuels (diversité, autonomie). Laborie (2006) définit l'activité de prise de décision collaborative comme: "une convergence d'interactions cognitives et visuelles, planifiées ou opportunistes, où des personnes acceptent de se rassembler pour un objectif commun, dans une période de temps définie, soit au même endroit, soit dans des endroits différents, dans le but de prendre des décisions».

La distinction entre la décision individuelle, collective, collaborative est, ainsi, principalement fondée sur deux éléments : la multiplicité des décisions individuelles et le partage d'intérêt. Ce partage d'intérêt du groupe est important. Il révèle un point essentiel qui différencie la décision en face à face et à distance : la motivation individuelle (Deci et Ryan, 1985) et l'envie de partager et de participer (Mansor et al., 2015). La décision collective est dite «imposée» par Novak et Ulfarino (2017). Par contre, en focalisant sur l'envie, une émotion sociale (Damasio, 2003), le groupe collaboratif ne décidera ensemble que si chacun en trouve la motivation, l'intérêt personnel.

4. Collective ou collaborative : contrairement au collaboratif, les efforts et décisions collectives sont l'agrégation des efforts individuels des gens, parfois dans le même service, mais ne partagent pas automatiquement un intérêt commun. Les deux types de décisions sont existantes à distance, par exemple, les clients qui aident à l'amélioration de la qualité d'un produit, n'attendent pas de bénéfice direct. 
En cela, la définition de la décision collaborative à distance sur laquelle nous appuyons notre investigation est : Différenciées des décisions collectives, les décisions collaboratives à distance sont l'agrégation de décisions individuelles d'individus autonomes qui, s'ils partagent un but commun, ne recherchent pas automatiquement un même intérêt, ni une même motivation. Elles reposent sur une émotion sociale qui favorise le partage dans une période de temps définie et un espace indéfini grâce à l'usage de technologies.

Un des éléments clés est celui du passage d'une collection de décisions particulières à une décision collaborative. Ainsi, pour obtenir un maximum d'éléments étant donné le manque d'études sur la décision collaborative à distance, une revue autour des catégories de décision en comparaison est réalisée.

\section{Comparaison des prises de décision}

Cette revue des comparaisons, effectuées entre la décision individuelle et collective puis les décisions collectives en face à face et collaboratives à distance, permet d'appuyer les effets de chacune des catégories de décision. Chacun de ces effets révèle à la fois des distinctions et quelques biais sous-jacents. Le tableau 1 classe les études de comparaison individuel/collectif par thème et date de parution, puis les études de comparaison collectif « en face à face” et collaboratif «à distance» en fonction des thèmes du premier classement.

Nous pouvons retenir du tableau 1 plusieurs spécificités de la décision collective d'une part :

1. elle permet la détection des erreurs, la meilleure compréhension des problèmes et le partage d'informations,

2. elle est inhérente à l'organisation complexe,

3. elle demande une compréhension processuelle et séquentielle,

4. à cause d'une stratégie collective probable, elle ne garantit pas la prise de décision optimale et l'impartialité des jugements, mais tend à diminuer la prise de risque

5. malgré l'apparent manque de consensus des résultats sur le biais de cadrage ${ }^{5}$, elle est soumise à certains biais individuels et n'en corrige pas les effets négatifs, lorsqu'elle ne l'augmente pas (escalade d'engagement)

6. elle est soumise à des biais, dits collectifs, tels la stratégie de groupe.

Les points (5 et 6 ) montrent, malgré les divergences dans certains résultats obtenus liés au type d'études et à leur protocole, que la décision de groupe peut s'avérer être un cumul des biais collectifs et individuels.

Les études de la décision collaborative à distance indiquent, quant à elles que, reposant sur des bases similaires à la décision collective, celle-ci n'est pas plus favorable à la rationalité optimale. De plus, elle perturbe les relations interpersonnelles, non seulement en permettant moins d'inhibition avec la possibilité d'exacerber ses émotions, particulièrement sous anonymat. Pour exemple, Helmersen et al. (2001) parlent de débordement et d'attracteur cognitifs dans la communication à distance par email. Lorsque, trop d'informations sont reçues. Certains mails qui se révèlent plus attractifs (urgents) que d'autres peuvent perturber la concentration nécessaire pour répondre aux messages qui demandent de la réflexion pour des décisions plus complexes. Turkle (1995), quant à lui, analyse les interactions lors de forums de discussion et prouve que l'intensité affective des échanges atteint des niveaux souvent tout à fait remarquables, exigeant dans certains cas l'application de règles éthiques par des administrateurs.

Ceci indique que la décision collaborative à distance repose sur un ensemble d'heuristiques - méthodes approximatives utilisées pour prendre une décision, telle une opération mentale rapide et intuitive- et de biais liés aux relations sociales interindividuelles, issues d'émotions sociales.

Afin de mieux comprendre comment limiter ses effets perturbateurs, toujours en considération du manque d'études sur la décision collaborative à distance, il est, à présent, question de faire un point spécifique sur les biais établis de ces trois catégories de décision, ce que Gautier (2015) nomme «l'erreur cognitive».

\section{Les biais liés à la décision individuelle/ collective/collaborative}

\section{LES BIAIS INDIVIDUELS ET COLLECTIFS}

Depuis de nombreuses années, plusieurs recherches, notamment en psychologie, ont mis en évidence une multitude de biais individuels de prise de décision. Lebraty et Pastorelli-Nègre (2004) en établissent une liste non exhaustive et décrivent chacun d'eux. Au niveau collectif, d'autres biais, moins nombreux s'ajoutent.

Le tableau descriptif suivant (tableau 2) nous permet d'en établir une classification selon leur degré émotionnel ou cognitif, un axe qui nous apparait un indicateur dichotomique révélateur de différenciation. Outre le fait qu'elle a une place proéminente dans la décision collaborative à distance, l'émotion est un facteur de prise de décision (Damasio, 1995, 2000). Elle se manifeste tant dans les biais que dans la décision. Ainsi, l'émotion positive, telle que la joie, est un facteur de bonne prise de décision (Isen, 1984), alors que l'émotion négative est un facteur de mauvais jugement -aversion, peur des risques- (Kahneman et Tversky, 1984). Etant donné que Damasio (2000) suggère que la décision repose sur une « régulation homéostasique» émotion-cognition, la distinction par l'émotion/cognition va aider à la construction d'une stratégie de réduction de biais de la décision collaborative à distance (cf. tableau 2).

$\mathrm{Au}$ vu du tableau 2, l'aspect cognitif est particulièrement prégnant dans les biais individuels, alors que l'émotionnel est essentiellement ancré au sein des biais de groupe. Ce constat permet d'expliquer en quoi les biais individuels peuvent avoir été analysés comme démultipliés lors de prises de décisions de groupe (tableau 1). L'émotion possède, en effet, une faculté de contagiosité indéniable (Hatfield et al, 1994) menant à une contagion émo-décisionnelle (van Hoorebeke, 2008). Ceci pourrait expliquer, pour partie, comment les dirigeants de Kodak -entreprise spécialisée dans les pellicules argentiques-, appuyés par des conseils d'administrations et une armée de consultants et d'experts, ont pris la décision qui fut fâcheuse pour l'entreprise d'ignorer certaines opportunités technologiques ${ }^{6}$.

5. fonction du cadre de la décision. Ce cadre est soumis à l'influence de la manière dont un problème est formulé, des valeurs, habitudes et préférences du décideur. 6. https://creg.ac-versailles.fr/IMG/pdf/article_decision.pdf 
TABLEAU 1

Etudes et réflexions comparatives de la prise de décision collective/individuelle et collective à distance

\begin{tabular}{|c|c|c|}
\hline Thème & Prise de décision collective / prise de décision individuelle & $\begin{array}{l}\text { Prise de décision en face à face / } \\
\text { prise de décision à distance }\end{array}$ \\
\hline Prise de risque & $\begin{array}{l}\text { - Les participants prennent significativement moins de risques lorsqu'ils sont en groupe (Masclet } \\
\text { et al. 2009) }\end{array}$ & \\
\hline Confiance et réciprocité & $\begin{array}{l}\text { - Les représentants de groupe montrent moins de confiance et de réciprocité avec le groupe que } \\
\text { les membres du groupe entre eux. (Song, 2008) }\end{array}$ & $\begin{array}{l}\text { - Relations sociales sources d'insatisfaction et } \\
\text { de frustration (Siegel et al. 1986) }\end{array}$ \\
\hline \multirow[t]{2}{*}{ Performance } & $\begin{array}{l}\text { - Le simple fait d'être membre d'un groupe change le choix individuel initial. (Kocher et Sutter 2007) } \\
\text { - } 60 \% \text { des participants ont choisi de décider collectivement. Les équipes ont été significativement } \\
\text { meilleures que les individus seuls. (Kocher et al. 2004) }\end{array}$ & $\begin{array}{l}\text { - Performance fonctionnelle de groupe est } \\
\text { montrée moins efficace. Le groupe fonctionne } \\
\text { de façon plus désordonnée (Strauss, 1996; Balaji } \\
\text { et Chakrabarti 2010) }\end{array}$ \\
\hline & $\begin{array}{l}\text { - Les conditions d'une action collective peuvent être différentes, voire même opposées aux } \\
\text { conditions d'une «bonne» décision collective. (Brunsson, 1982) }\end{array}$ & $\begin{array}{l}\text { - Moins de pression de performance et plus } \\
\text { de comportements débridés sous anonymat } \\
\text { (critiques désinhibées) et favorise la pensée } \\
\text { divergente (Connolly, Jessup et Valacich, 1990; } \\
\text { O'Reilly et Roberts, 1977) }\end{array}$ \\
\hline \multirow{3}{*}{$\begin{array}{l}\text { Choix rationnel } \\
\text { La théorie du choix rationnel } \\
\text { suppose de comprendre les } \\
\text { phénomènes sociaux au travers } \\
\text { des actions individuelles et de leur } \\
\text { donner du sens. (Boudon, 2002) }\end{array}$} & $\begin{array}{l}\text { - Les membres du groupe corrigent mutuellement leurs erreurs et mettent en commun des } \\
\text { ressources complémentaires (connaissances, compétences, etc.) afin de résoudre collectivement } \\
\text { des problèmes qu'aucun ne saurait résoudre seul (Stasser et Dietz-Uhler, 2001) }\end{array}$ & \\
\hline & $\begin{array}{l}\text { - Question de recherche : le groupe est-il plus rationnel que l'individu? } \\
\text { - La théorie du choix rationnel utilisée dans leur étude ne permet pas de distinguer les choix du } \\
\text { groupe par rapport à ceux des individus, considérant que les membres d'un groupe peuvent } \\
\text { réaliser un agrément contraint d'une stratégie collective. (Bornstein et al., 2004) }\end{array}$ & $\begin{array}{l}\text { - Choix selon le mérite de l'idée plutôt que par } \\
\text { rapport au statut de la personne (Jessup, } \\
\text { Connolly et Galegher, 1990) }\end{array}$ \\
\hline & $\begin{array}{l}\text { - Le groupe se comporte de façon quasi identique d'un point de vue «rationalité » et «confiance» } \\
\text { que les individus isolés. (Kugler et al. 2012) }\end{array}$ & \\
\hline \multirow[t]{3}{*}{$\begin{array}{l}\text { Effet de cadrage (biais lié à la formu- } \\
\text { lation du choix, positive ou négative) }\end{array}$} & $\begin{array}{l}\text { - L'effet de cadrage est réduit lors de décisions de groupe par rapport aux décisions prises de } \\
\text { façon individuelle. (Neale et al. 1986) }\end{array}$ & \\
\hline & $\begin{array}{l}\text { - L'effet de cadrage est supérieur pour des décisions de groupes homogènes. Lorsque la même } \\
\text { formulation est présentée à des sujets, à la fois individuellement et en groupe, les effets de } \\
\text { cadrage au niveau individuel sont plus élevés qu'en groupe. Lorsque la formulation opposée est } \\
\text { présentée aux groupes, les effets de cadrage initiaux sont réduits. (Paese et al.1993) }\end{array}$ & \\
\hline & $\begin{array}{l}\text { - L'effet de cadrage n'est pas significativement supérieur ou moindre lors de décisions de groupe. } \\
\text { Les groupes ne répondent pas de la même manière que les individus à l'encadrement des } \\
\text { options de choix, mais le choix des groupes dépend d'une constellation de facteurs tels que les } \\
\text { caractéristiques de la décision elle-même. (Milch, Weber et al. 2009) } \\
\text { - La comparaison des choix du groupe hétérogène et des choix individuels montre que l'effet de } \\
\text { cadrage est amplifié au niveau du groupe hétérogène. Le groupe dit hétérogène est composé } \\
\text { de personnes confrontées pour moitié à un problème à résoudre en termes positifs (gagner de } \\
\text { l'argent) et l'autre moitié en termes négatifs (ne pas perdre d'argent). (Yaniv, 2011) }\end{array}$ & \\
\hline
\end{tabular}




\section{TABLEAU 1}

Etudes et réflexions comparatives de la prise de décision collective/individuelle et collective à distance

\section{Thème}

Prise de décision collective / prise de décision individuelle

Prise de décision en face à face $/$

Escalade d'engagement. Il s'agit

d'un biais par l'engrenage qu'il

provoque. L'engagement crée

une consolidation des décisions

et attitudes et de nouveaux

comportements. (Lewin, 1952)

\begin{tabular}{l|l} 
Processus & - Au sein des organisations, le processus de décision ne peut être compris à partir d'un seul acteur,
\end{tabular}

de manière individuelle. Le processus de décision y est collectif et séquentiel. (Allison, 1971)

\begin{tabular}{l|l} 
Processus complexe & - Les processus de décision dans les organisations impliquent généralement plusieurs acteurs
\end{tabular} interagissant les uns avec les autres. (Smoliar et Sprague, 2002)

- Le groupe à distance prend plus temps pour décider 4 à 10 minutes de plus (Reid, Ball, Morley et Evans, 1997)

Et 5 à 6 fois plus de temps pour trouver un consensus que le groupe en face à face (Walther 1996 \begin{tabular}{|l|l} 
Complexité & $\begin{array}{l}\text { Plus les organisations deviennent complexes, moins les décisions sont prises par des individus } \\
\text { seuls. (Keen et Scott-Morton, 1978) }\end{array}$
\end{tabular}

- Le groupe a distance permet la prise décision pa de grands groupes (DeSanctis et Gallupe, 1987)

Common knowledge, partage

- Un élément d'information aura plus d'influence sur la décision lorsqu'il est partagé que quand

d'informations il est ne l'est pas. (Stasser et al. 1989)

Partage d'informations - La prise de décision collaborative a les avantages suivants sur la décision individuelle :

- les erreurs sont mieux saisies et détectées

- les problèmes bien compris et une plus grande quantité d'information et de connaissances est fournie. (Turban et al., 2001)

Le groupe est moins conforme et a moins d'inhibition à exprimer ses idées qu'en face à face (El-Shinnawy et Vinze, 1997) 
TABLEAU 2

Les biais individuels et collectifs

\begin{tabular}{|c|c|c|c|c|c|}
\hline Biais individuels & Description & Biais collectifs & Description & $\begin{array}{l}\text { Description du choix de } \\
\text { la catégorie selon les } \\
\text { verbatims }\end{array}$ & Emotion/ cognition \\
\hline $\begin{array}{l}\text { Loi des nombres pairs } \\
\text { (Schwenk, 1989) }\end{array}$ & $\begin{array}{l}\text { - Utilisation d'un petit nombre d'informations. Risque } \\
\text { d'élimination d'informations pertinentes }\end{array}$ & & & Informations éliminées & Biais cognitif \\
\hline $\begin{array}{l}\text { - Illusion de pointage } \\
\text { - Similarité et fréquence } \\
\text { - Confirmation } \\
\text { - Illusion de contrôle } \\
\text { - Ajustement apparent } \\
\text { (Reason, 1993) }\end{array}$ & $\begin{array}{l}\text { - Vérification de la liste des facteurs à considérer est } \\
\text { rarement complète } \\
\text { - Dans le cadre de tâches répétitives, certaines } \\
\text { erreurs sont difficilement détectables en raison de } \\
\text { leur similarité ou fréquence } \\
\text { - Face à une ambiguïté, faveur pour une interprétation } \\
\text { disponible } \\
\text { - Croyance selon laquelle on peut avoir un contrôle sur } \\
\text { certains résultats } \\
\text { - Un modèle, même peu conforme à la réalité, ne fait } \\
\text { pas forcément apparaître d'éléments susceptibles } \\
\text { de l'infirmer }\end{array}$ & & & $\begin{array}{l}\text { informations, vérifications, } \\
\text { contrôle, interprétations, } \\
\text { infirmer }\end{array}$ & Biais cognitifs \\
\hline $\begin{array}{l}\text { Rétrospection } \\
\text { (Fischoff, 1975) }\end{array}$ & $\begin{array}{l}\text { - La connaissance du résultat final par le décideur } \\
\text { influence fortement la façon dont on reconstruit } \\
\text { l'enchaînement des décisions associées }\end{array}$ & & & & Biais cognitif \\
\hline $\begin{array}{l}\text { Coûts immobilisés } \\
\text { (Arkes et Blumer, 1985) }\end{array}$ & $\begin{array}{l}\text { - Les décisions de poursuivre un investissement se font } \\
\text { sans tenir compte des sommes déjà immobilisées }\end{array}$ & & & connaissance, tenir compte & Biais cognitif \\
\hline $\begin{array}{l}\text { Disponibilité de mémoire } \\
\text { (Hogarth et Einborn, 1992) }\end{array}$ & $\begin{array}{l}\text { - les décideurs privilégient les mécanismes } \\
\text { heuristiques et les informations les plus récemment } \\
\text { mis en mémoire }\end{array}$ & & & mémoire, informations & Biais cognitif \\
\hline $\begin{array}{l}\text { Effet d'ancrage } \\
\text { (Tversky et Kanheman, 1974) }\end{array}$ & $\begin{array}{l}\text { - attribuer une pondération excessive à une information } \\
\text { fonction du cadre décisionnel, i.e. de l'influence de } \\
\text { la manière dont un problème est formulé, de la part } \\
\text { des valeurs, habitudes et préférences du décideur }\end{array}$ & & & informations & Biais cognitif \\
\hline $\begin{array}{l}\text { Effet de cadrage } \\
\text { (Kanheman et Tversky, 1984) }\end{array}$ & & & & & Biais cognitif \\
\hline $\begin{array}{l}\text { Corrélations illusoires } \\
\text { (Rai et al., 1994) }\end{array}$ & $\begin{array}{l}\text { - Lorsque deux événements sont corrélés, attribuer à } \\
\text { l'un d'eux des relations de cause à effet }\end{array}$ & & & corréler, attribuer & Biais cognitif \\
\hline $\begin{array}{l}\text { Excès de confiance } \\
\text { (Roy et Lerch, 1996) }\end{array}$ & $\begin{array}{l}\text { - accorder à une connaissance une exhaustivité et une } \\
\text { précision qu'elle n'a pas }\end{array}$ & $\begin{array}{l}\text { Illusion de } \\
\text { l'introspection } \\
\text { Nisbett et al. } \\
\text { (1977) }\end{array}$ & $\begin{array}{l}\text { Effet de contamination : } \\
\text { interprétation et } \\
\text { perception sélective } \\
\text { d'informations allant dans } \\
\text { le sens d'une première } \\
\text { impression que l'on } \\
\text { cherche à confirmer }\end{array}$ & connaissance & $\begin{array}{l}\text { Biais cognitif: } \\
\text { sélection des } \\
\text { informations } \\
\text { selon la première } \\
\text { impression, } \\
\text { perception }\end{array}$ \\
\hline
\end{tabular}


TABLEAU 2

Les biais individuels et collectifs

\begin{tabular}{|c|c|c|c|c|c|}
\hline Biais individuels & Description & Biais collectifs & Description & $\begin{array}{l}\text { Description du choix de } \\
\text { la catégorie selon les } \\
\text { verbatims }\end{array}$ & Emotion/ cognition \\
\hline $\begin{array}{l}\text { Conformité au groupe } \\
\text { Helmreich et Meritt (1998) }\end{array}$ & $\begin{array}{l}\text { - Favoriser les décisions qui maintiennent la cohésion } \\
\text { de groupe }\end{array}$ & $\begin{array}{l}\text { Pensée de } \\
\text { groupe } \\
\text { (Janis, 1982) }\end{array}$ & $\begin{array}{l}\text { Conformisme, Effort pour } \\
\text { établir un consensus aux } \\
\text { dépens d'une évaluation } \\
\text { réaliste des alternatives }\end{array}$ & $\begin{array}{l}\text { conformisme, recherche de } \\
\text { cohésion, mais effort pour } \\
\text { établir un consensus }\end{array}$ & $\begin{array}{l}\text { Biais } \\
\text { émotionnel et } \\
\text { cognitif }\end{array}$ \\
\hline $\begin{array}{l}\text { Reprise de contrôle } \\
\text { (Pastorelli, 2000). }\end{array}$ & $\begin{array}{l}\text { - Favoriser les informations pour lesquelles une } \\
\text { intervention de leur part est nécessaire, même si } \\
\text { celle-ci est inappropriée }\end{array}$ & & & contrôle, informations. & Biais cognitif \\
\hline \multicolumn{6}{|l|}{$\begin{array}{l}\text { Biais individuels spécifiques } \\
\text { aux décisions médiées «par } \\
\text { ordinateur» }\end{array}$} \\
\hline $\begin{array}{l}\text { Création d'attentes } \\
\text { (Klein, 1998) }\end{array}$ & $\begin{array}{l}\text { - Le SAD peut distordre le contexte général d'une } \\
\text { situation, et contribuer à mettre les utilisateurs dans } \\
\text { un contexte qui crée des attentes liée à son influence }\end{array}$ & $\begin{array}{l}\text { Biais du } \\
\text { champion } \\
\text { Lefley (2006) }\end{array}$ & $\begin{array}{l}\text { Evaluation basée } \\
\text { sur l'expérience et } \\
\text { la compétence de la } \\
\text { personne qui présente } \\
\text { plutôt que sur les faits }\end{array}$ & $\begin{array}{l}\text { l'expérience ou expertise } \\
\text { perçue de la personne ou } \\
\text { de l'ordinateur influence } \\
\text { la décision }\end{array}$ & Biais émotionnel \\
\hline \multirow[t]{3}{*}{$\begin{array}{l}\text { Automation } \\
\text { (Skitka et al., 1999) }\end{array}$} & $\begin{array}{l}\text { - Laisser travailler le Système d'aide à la décision (SAD) } \\
\text { en leader }\end{array}$ & $\begin{array}{l}\text { Management } \\
\text { du tournesol } \\
\text { Boot et al. } \\
\text { (2005) }\end{array}$ & $\begin{array}{l}\text { Tendance assumée à } \\
\text { s'aligner sur la vision } \\
\text { du leader }\end{array}$ & $\begin{array}{l}\text { le leadership influence } \\
\text { la décision }\end{array}$ & Biais émotionnel \\
\hline & & $\begin{array}{l}\text { Biais spécifique } \\
\text { à la décision } \\
\text { collective à } \\
\text { distance }\end{array}$ & & & \\
\hline & & $\begin{array}{l}\text { Deindividuation } \\
\text { Lea et Spears } \\
\text { (1992) }\end{array}$ & $\begin{array}{l}\text { Désorganisation du } \\
\text { groupe, liée aux liens } \\
\text { interpersonnels faibles } \\
\text { ou aux comportements } \\
\text { inappropriés surtout } \\
\text { sous anonymat }\end{array}$ & $\begin{array}{l}\text { manque de relations } \\
\text { sociales, de contacts } \\
\text { interindividuels }\end{array}$ & Biais émotionnel \\
\hline
\end{tabular}

Tiré de Lebraty et Pastorelli-Nègre (2004)

Légende : pour catégoriser les biais, une analyse de contenu manuelle a été réalisée selon les verbatim définissant chaque biais

Au niveau individuel, les biais sont surtout cognitifs.

Seul un biais classé cognitif, excès de confiance (ind.) et illusion d'introspection (coll.), est à la fois valable au niveau individuel que collectif.

A part le conformisme, lié à une volonté et un effort pour trouver un consensus, les biais collectifs sont essentiellement liés à l'émotionnel.

Le biais de deindividuation est le seul biais connu spécifique à la décision collective à distance. Il est analysé comme émotionnel. Il est issu de la psychologie des foules et correspond à une mise à l'écart de la conscience de soi par le fait de se sentir affilié à un groupe. ${ }^{16}$ 


\section{LES BIAIS DE LA DÉCISION COLLABORATIVE À DISTANCE}

Les biais de la prise de décision collaborative font moins l'objet d'études. Néanmoins, Stasser et Stewart (1992) mettent en exergue le biais de discussion, ou de sélection des informations à partager pour suivre la stratégie voulue. Stasser et Titus (1985) parlent de "common information sampling bias". Certaines informations connues par plusieurs membres du groupe seront davantage discutées que les informations connues d'un seul membre. Ces deux biais reposent sur le fait que les discussions à distance sont moins développées et sont davantage centrées sur l'objet principal. On y évite les discussions vues comme superflues qui ne seraient pas éludées en face à face (Herring, 1996). Zigurs et al. (1988) montrent, quant à eux, qu'il existe certains points de différence significative au sujet de l'influence sociale pour les groupes à distance. A ce sujet, Dubrovsky (1985) précise que la distance par ordinateur favorise la rationalité en permettant de se focaliser sur l'essentiel de la tâche à réaliser en filtrant les composantes affectives de la communication, en minimisant l'influence sociale liée au statut et aux «bruits» interpersonnels. En complément, Lea et Spears (1991) montrent que la «deindividuation ${ }^{7}$ ", existante dans le cadre de la décision à distance avec perception d'anonymat, conduit à des comportements qui ne seraient pas acceptés en situation de face à face. Ainsi, l'anonymat serait particulièrement favorable au dévoilement émotionnel, car les participants peuvent être plus distanciés par rapport au jugement possible d'autrui. Ce type d'interaction est qualifiée d'"hyperpersonnelle» par Walther (1996). Face à cette distanciation, des études montrent que l'individu va chercher à recréer une relation sociale par des méthodes différentes de celles utilisées en face à face (les écrits, images...) (Reid, 1991). Ainsi, à long terme, les individus créent des relations sociales entre eux et, souvent, faute de se rencontrer physiquement, imaginent leur interlocuteur de façon stéréotypée (Lea et Spears, 1991). Ceci induit un biais d'auto-représentation (Lea et Spears, 1992). L'individu se montre dans un meilleur aspect qu'il ne le serait en face à face et peut utiliser une communication plus excessive. Cela pénalise le consensus qui réclame des échanges sociaux fondés sur un édifice moins superficiel (Walther, 1996; Siegel et al., 1986), mieux organisé (Rice, 1984), autour d'un leadership.

Comme l'indiquent Baltes et al. (2002), la plupart de ces études date d'années où la technologie n'était pas aussi évoluée que de nos jours. Néanmoins, il est à envisager que les résultats décrits ne laissent pas entrevoir de biais fondamentalement différents de la prise de décision en face à face, à part sous anonymat où le biais du statut de la personne n'a plus d'effet (Dubrovsky et al. 2009) où les émotions sont libérées et où la pensée divergente est admise, réduisant le biais de la pensée unique. Puisque les stratégies de réduction de biais dans la littérature sur la décision collaborative à distance sont elles aussi insuffisantes, une revue des stratégies de réduction de biais de la prise de décision des trois catégories de prise de décision va nous être, ici aussi, nécessaire.

\section{Vers une nouvelle stratégie de "debiasing" de la décision collaborative}

\section{LES STRATÉGIES DE RÉDUCTION DE BIAIS ACTUELLES}

La littérature anglo-saxonne met en relief le concept de «debiasing». Ce concept porte sur les moyens qui permettent de limiter l'effet des biais décisionnels. L'article de Soll et al. (2013) propose un guide pour limiter les biais décisionnels individuels. Pour résumer, leur guide obtenu par une méta-analyse énumère les éléments à maîtriser, tels que "convertir» le décideur par l'éducation et divers outils d'aide à la décision; ou modeler l'environnement en créant les conditions optimales au bon jugement (Soll et al., 2013). Lerner et al. (2015) suggèrent de modeler méticuleusement l'architecture du choix pour limiter les biais. Selon le modèle de Wilson et Brekke (2002), réalisé à partir d'une illustration descriptive, la réduction de biais suit un processus cognitif, à l'instar de la décision. Dans un premier temps, l'individu doit être motivé à corriger l'erreur. Pour cela, l'individu doit (1) comprendre que son impression est biaisée, (2) souhaiter qu'elle soit modifiée, selon son appréciation de l'effort demandé pour la corriger. Deuxièmement, il lui faut pouvoir être conscient du degré et de la magnitude du biais et de ses conséquences. Troisièmement, un contrôle suffisant sur la décision prise est nécessaire pour pouvoir la corriger. (Wilson et al., 2002, p.186).

Kennedy (1993) montre l'influence positive du processus de révision par d'autres individus, comme outil de réduction des biais de jugements comptables. A ce sujet, Kahneman (2003) explique que l'individu seul ne peut limiter les biais. Larrick (2004) affirme, lui aussi, que la réduction des biais nécessite une intervention extérieure. Du même avis que Wilson et al. (2002), Larrick (2004) confirme néanmoins, que l'individu doit tout d'abord avoir conscience du biais. Selon lui, la difficulté de cette étape prouve que les outils de réduction de biais sont importants à étudier pour améliorer les prises de décisions futures, notamment les stratégies dont doivent être équipés les décideurs. Cet auteur réalise, à ce sujet, un répertoire des stratégies de réduction de biais, selon la motivation, le technologique et le cognitif. Au vu de ces écrits, les statistiques et la raison (cognition) tiennent une large part dans les dispositifs de réduction de biais individuels grâce aux analyses de données et algorithmes. Pourtant dès 1987, Gigerenzer et Murray ajoutent à cette vision la neurologie et les stratégies psychologiques au travers de l'heuristique. Selon cette vision, la décision est modélisée à partir de processus cognitifs et non uniquement à partir du formel et de l'arithmétique.

C'est cette dimension heuristique, cognitive individuelle et de relation sociale, composée de croyances, d'émotions et d'intentions, envisagée dans les groupes de décision, qui nous mène à la réflexion d'un levier de réduction de biais. Nous nous focalisons sur les émotions. Elles sont, non seulement, vues comme partie intégrante de la décision de groupe et de ses biais -comme montré en tableau 2-, mais aussi nécessaires à la décision à distance pour éviter l'insatisfaction liée au manque de relations sociales. De plus, elles sont universelles, i.e. similaires quelle que soit la culture (Ekman et Oster, 1979) ce qui peut favoriser le consensus malgré la diversité des membres du groupe à distance.

\section{LES ÉMOTIONS, FACTEURS DE BIAIS ET DE RÉDUCTION DE BIAIS}

Lorsqu'elles sont évoquées comme influentes de la décision, c'est souvent l'état émotionnel de l'individu qui est étudié, comme l'ont démontré plusieurs recherches (voir pour revue,

7. Mise à l'écart de la conscience de soi par le fait de se sentir affilié à un groupe, déshumanisation. 
Lerner et al. 2015). Comme évoqué plus avant, l'émotion peut être appréciée à la fois comme un facteur facilitateur de la décision et un facteur de biais. Par exemple, Lacroix (2014) montre, par l'étude d'interventions de marins-pompiers, que la décision est soumise à ce dilemme émotionnel. Elle peut être à la fois perturbée par les émotions négatives -liées au secours d'un membre de la famille- et un appui à la décision par les émotions positives de cohésion de groupe. En tant que facteur de biais de la prise de décisions, l'état émotionnel peut affecter le contenu de l'information et modifier un jugement (LeDoux, 1993). L'émotion négative intense peut induire un jugement biaisé. Un choix à court-terme sera, dans ce cas, perçu plus plaisant qu'un choix à long-terme (Gray, 1999). A contrario, l'émotion peut faciliter la performance décisionnelle par sa faculté à stimuler l'attention consciente et la mémoire nécessaire au raisonnement et à la décision (hypothèse du marqueur somatique, Damasio, 1995). L'émotion agréable ou désagréable soutient, ainsi, les décideurs à résoudre le dilemme du choix face aux différentes options ou aux avantages contradictoires en s'appuyant sur un ressenti distinctif (Raghunathan et Pham, 1999). A ce sujet, Schwarz et Clore (2003) précisent que l'émotion aide au choix par une sélection affective des priorités adaptées à une situation donnée. Un exemple ordinaire: les quelques fois où j'ai eu ce collègue par mail, il a été odieux. Au moment de le contacter, je ressens une impression désagréable. J'ai du mal à me décider à le contacter.

\section{Les émotions outils de réduction de biais de la décision collaborative à distance}

Pour aller plus avant, les résultats de l'étude en neurologie de De Martino et al. (2006), à propos du biais décisionnel de l'effet de cadrage -manipulation de la formulation du choix- montrent que le biais est limité lorsque l'émotion est gérée ou mise en équilibration dynamique avec la cognition. Cette recherche d'équilibre est appuyée par LeDoux (2000, p.157) «Minds are not either cognitive or emotional, they are both, and more», et Kahneman (2011) dans son article "Thinking, Fast and Slow». Ce dernier précise que notre pensée est composée de deux systèmes en interaction. Le premier est décrit comme inconscient, il peut s'apparenté à l'émotionnel. Il est intuitif, naturel et très rapide. Il l'a nommé «fast». Le second, nommé «slow», est mis en fonctionnement par le premier lorsque nécessaire. Il représente la réflexion, le raisonnement, soit l'aspect cognitif. Le premier est, selon lui, porteur de biais, le second a la faculté de corriger ces biais s'il y est entrainé.

Une question se pose, dans la décision collaborative à distance, l'émotionnel doit-il être régulé d'une façon similaire? En effet, certaines tâches demandent des échanges sociaux qui peuvent être perturbés par la relation par ordinateur (Strauss et McGrath,1984). Selon les résultats de Derks Fischer et Bos (2007), la décision collaborative à distance repose à la fois sur une fréquence plus marquée et plus explicite de l'émotionnel et sur une spontanéité plus réduite, liée à un contrôle plus développé. Svensson (2014) parle, à ce sujet, d'un management des émotions renforcé pour marquer son image, son statut, voire son pouvoir. La prise de décision collaborative à distance apparait, ainsi, plus qu'appropriée à être régulée. En cela, ce type de décision favoriserait l'équilibration émotion/cognition, source de réduction de biais (LeDoux, 2000; Kahneman, 2011). Cette dernière synthèse théorique nous conduit, ainsi, à établir une première proposition de recherche :

Proposition 1 : Grâce au paradoxe qu'elle connaît -spontanéité limitée/explicité de l'émotionnel-, la décision collaborative à distance peut reposer sur un processus dynamique d'équilibration émotion/cognition réducteur de biais.

\section{Un processus à la fois cognitif et émotionnel pour réduire les biais}

En réponse à l'atteinte de cet équilibre, il est considéré, ici, que l'individu effectue un travail émotionnel. Celui-ci est, en effet, indispensable dans les relations sociales au travail, y compris sur internet (Svensson, 2014), et pour pouvoir décider à travers une régulation des émotions (Grandey, 2003). Nous pouvons, ainsi, proposer un processus de réduction des biais. Ce processus allie celui de Wilson et al. (2002), décrit ci-avant, qui détaille les étapes cognitives, et la théorie de Kahneman (2011), qui explique le système d'équilibre émotion/cognition au sein de la décision «fast and slow». Le processus suggéré repose sur plusieurs étapes :

1. comme le suggère Wilson et al. (2002), il faut dans un premier temps, une prise de conscience du biais, une volonté de le corriger et une évaluation de son degré et de sa magnitude. Cette première étape équivaut à l'usage du système 2 , «slow» de Kahneman, qui y aura été préalablement entrainé.

2. Grâce à cette prise de conscience, un processus émotionnel est déclenché. Si l'individu ressent une émotion, issue du système 1 , qui diffère de la décision qu'il a exprimée (Abraham, 1998), il ressent une dissonance émotionnelle. Pour corriger cette dissonance, l'individu va devoir effectuer un effort cognitif, le travail émotionnel.

3. le travail émotionnel (Hochschild, 1979) correspond à un double effort (émotion/cognition) fourni par le décideur pour maitriser ses émotions dans le sens voulu vers une décision optimale. Il est composé de deux types de stratégies, le travail en surface (surface acting) et en profondeur (deep acting). Le travail en surface va inciter l'individu à exprimer sa dissonance sans modifier son ressenti, par exemple : je préfère me taire ou exprimer mon désaccord. Le travail en profondeur va l'aider à mieux analyser sa prise de décision pour éviter tout biais. Par exemple : suis-je en désaccord parce que cette décision me rappelle une mauvaise expérience? ou fait montre d'une pensée unique? La seconde stratégie cherche, ainsi, à modifier et adapter le ressenti par une régulation en profondeur consciente (Grandey, 2000) (Système 2 de Kanheman).

De façon collaborative pour une recherche de consensus, chaque membre du groupe individuellement suit ce processus. Dans le cadre d'une discussion interindividuelle à distance, chaque individu a la possibilité d'exprimer sa dissonance (surface acting) et prendre du recul par rapport à la discussion (attendre avant d'intervenir et se mettre en retrait momentanément d'une décision). Cette expression permet à chacun de repérer un biais et, par sa prise de distance, de modifier son ressenti ou trouver une stratégie pour alerter les autres de la 
présence d'un biais (deep acting). Le biais collaboratif peut, à ce moment, être corrigé.

Cette stratégie est proche (Larrick, 2004) de la norme attendue enseignée et adoptée. Elle utilise de façon positive les litiges (Babcock et al.,1997) y compris concurrentiels. Elle s'appuie, en cela, parfois sur des régularités qui favorisent une solution satisfaisante, adoptée par le plus grand nombre (Mione et Leroy, 2013). Elle est étayée par un double effort (cognitif et émotionnel, système 1 et 2 en interaction) fourni par chaque décideur pour limiter les biais de la prise de décision. Ce processus s'apparente à celui de Sechrist et Stangor (2001), pour qui le processus de recherche de consensus affiche en surface un rapprochement des perspectives, avec un élargissement des groupes de pensée, puis plus en profondeur, induit la saillance d'une dimension commune. Il n'a pas pour objectif de mener à un effet moutonnier et une pensée unique. Au contraire, il incite le groupe au travers de chacun de ses membres à développer un système de réflexion plus approfondi grâce à une régulation des émotions constitutif d'une heuristique ${ }^{9}$ de jugement appuyée par des débats constructifs, voire divergents. Par exemple, je sais pourquoi je n'aime pas cette décision, je peux, à présent en débattre.

Proposition 2 : Les stratégies du travail émotionnel de groupe (travail en surface et en profondeur) supportent la dissonance pour une prise de décision collaborative à distance limitée en biais.

\section{Les facteurs de ce processus de réduction de biais cognitivo- émotionnel}

Ce processus est appuyé par plusieurs facteurs. Une étude a cherché à assurer cette réduction de biais en parant le manque de relations sociales physiques par l'accompagnement. A partir d'une suggestion de Rice (1984), une recherche de Garrison et Cleveland-Innes (2005) en éducation online, étudie l'action de la structure (les tâches à réaliser, le design du site éducatif) et du leadership pour faciliter la présence cognitive. Ces deux axiomes pourraient être des facteurs de contingence favorables à la prise de décision collaborative à distance. Bien que leurs résultats montrent que ces facteurs ont un réel impact sur les relations sociales, ils ne sont pas vus par les auteurs comme suffisants. Nos propositions suivantes tendent à étudier ces mêmes facteurs, mais sous plusieurs dimensions managériales.

\section{LE FACTEUR STRUCTUREL}

Pour appuyer notre choix du facteur structurel, l'entreprise Cisco systems parle du collaboratif à distance comme d'une facilité à trouver les réponses aux questions, à localiser le spécialiste [...] accélérer la prise de décision sans quitter son poste, même si l'on travaille dans une entreprise d'envergure internationale ${ }^{10}$. La structure pourrait, selon nous, avoir pour première composante, le modèle circomplexe des tâches de groupe de McGrath (1984) qui dessine quatre types de tâches (Générer, Choisir, Négocier, Exécuter). La prise de décision (choisir) y est analysée comme une tâche où le groupe doit approuver une solution. Certaines décisions s'appuient sur les tâches décrites par Mintzberg et Waters, 1990 : (1) planification et créativité, (2) tâches intellectuelles et résolution de problèmes, (3) négocier -tâche sociale (Rice, 1984)- et (4) agir -la décision dépend de l'action-. Le travail émotionnel n'est pas adéquat dans toutes les tâches. Ainsi, s'il est pertinent lors de la prise de la prise de décision, il ne l'est pas dans l'exécution, résultat éventuel d'un travail émotionnel. De la même façon, le travail émotionnel, par son aspect inhibiteur et régulateur, peut pénaliser la créativité, incluse dans le modèle de Mintzberg et Waters (1990)

\section{LE FACTEUR SOCIAL}

Autre composante proposée par Lea et Spears, (1992) pour favoriser la dynamique de groupe et limiter l'effet de désindividualisation : le modèle SIDE ${ }^{11}$. Il mesure le système d'interactions du groupe par une "social self-categorization» (catégorisation sociale de soi), au travers de l'identité saillante du groupe et la coprésence des membres du groupe. La catégorisation sociale de soi est un facteur de liens entre les membres lorsqu'ils ne sont pas proches à l'origine. C'est aussi un facteur d'échanges "paralinguistiques' réducteurs d'incertitude et d'insatisfaction sociale. Cette composante s'appuie sur le fait que c'est effectivement le manque de relations socio-émotionnelles et personnelles (dynamique de groupe) qui induit l'échec à établir un consensus.

\section{LE FACTEUR DE RICHESSE DES MÉDIAS UTILISÉS}

Une troisième composante, le design. Selon Volery et Lord (2000), la distance est un moyen de communication plus riche que le face à face lorsqu'il cumule les médias utilisés pour communiquer et participer. Le cumul des médias est perçu comme un moyen de donner des expériences d'apprentissages différentes. C’est ce que Daft et Lengel (1986) nomment la théorie de la richesse média créant un environnement d'apprentissage permettant un feedback immédiat, des relations sociales, l'utilisation de messages différents et personnalisés. Selon une étude de MacKinsey (2012), les « collecticiels» CRM, messagerie instantanée, documents partagés..., en facilitant les interactions entre les équipes, favorisent l'intelligence collective. ${ }^{12}$

Proposition 3 : la structure (richesse des médias, type de tâche décisionnelle et SIDE model) favorise la réduction des biais de la décision collective à distance.

\section{LE FACTEUR LEADERSHIP}

Pour manager une équipe à distance, Skyrik (2010) suggère que le manager d'équipe devrait pouvoir disposer d'outils pour favoriser la dynamique de groupe. C'est le facteur structurel que nous venons d'exposer. Cisco systems ajoute le besoin d'un changement de mentalité des managers par un abandon du mode descendant (topdown) pour favoriser la ligne de front dans la décision ${ }^{13}$. Cela nous conduit au second facteur de contingence envisagé : le leadership. En effet, Darling-Hammond et Richardson (2009)

9. Mode de raisonnement

10. https://www.lesechos-etudes.fr/media/uploads/etudes/extrait/LEE_equipedispersee_HD.pdf

11. Le modèle SIDE repose sur l'effet de la désindividualisation sur le comportement du groupe. Ce modèle permet de mesurer le niveau de relations entre membres d'un groupe. Lea et Spears (1992) montrent, par ce modèle, que l'anonymat peut également polariser les petits groupes et devenir stratégique.

12. The social economy : Unlocking value and productivity through social technologies(McKinsey, 2012)

13. https://www.lesechos-etudes.fr/media/uploads/etudes/extrait/LEE_equipedispersee_HD.pdf 
prônent les capacités d'explication, d'enthousiasme et d'empathie del'instructeur, familières au leadership. Cet instructeur pourrait, ainsi, s'apparenter à un leader-accompagnateur dans la prise de décision à distance, capable d'organiser la prise de décision, de former aux biais, d'accompagner le travail émotionnel de groupe vers un consensus lié au partage d'opinions et d'aider à « concrétiser» l'existence virtuelle des autres membres.

Proposition 4 : la présence d'un leader-accompagnateur favorise la réduction des biais de la décision collective à distance

\section{Discussion}

Cette revue de littérature a, tout d'abord, permis d'effectuer un repérage des études qui confrontent la décision individuelle et la décision collective en face à face et à distance afin de découvrir (1) les différents biais attenants et (2) deux axes communs à ces biais et aux heuristiques : un axe cognitif et émotionnel, un axe temporel. Ces deux axes universels peuvent s'adapter à la diversité, notamment culturelle et de pratiques, de la prise de décision à distance, telle que par exemple, "les Japonais ont des procédés intéressants à ce sujet : nous avons fait des séances de travail [médiées] avec eux, et ils souhaitaient avoir un temps de débat entre eux, après chaque séquence. [...] nous n'avons pas l'habitude de ces pratiques. ». Ou encore, "dans notre culture, on a l'habitude du chef, alors que dans la démarche asiatique, celui qui écoute a aussi la capacité de comprendre comment chemine l'autre. Nous avons en Occident beaucoup de méthodes sur la prise de parole mais pas sur l'écoute» ${ }^{14}$.

Notre analyse montre que plusieurs éléments distinguent les biais de la décision collaborative à distance. Ceux-ci correspondent à une agrégation de décisions individuelles. Ils complètent les biais individuels et collectifs en face à face et possèdent des spécificités. Selon la littérature, la décision à distance n'est pas plus efficace, ni favorable à la rationalité et est soumise aux biais. Elle est insatisfaisante en termes de relations interpersonnelles défavorisant le consensus et est, à la fois, perçue très dépendante ou totalement dénuée de relations émotionnelles. Un exemple conforte ce manque d'humanisation, une startup est allée jusqu'à créer un système de reconnaissance faciale capable de saisir l'état émotionnel du collaborateur à distance ${ }^{15}$.

Les constats issus de revues de littérature (revue des comparaisons entre effets des décisions individuelles, collectives et à distance; des différents biais et de leurs stratégies de réduction de biais) ont permis d'aller plus avant et de détailler l'élément commun non étudié dans ce cadre jusqu'alors : l'émotion et son équilibration avec la cognition. Les heuristiques -fonctionnements mentaux- et les biais apparaissent indifféremment dans une ou plusieurs étape(s) du processus de la prise de décision, dépendantes entre elles. Il est, ainsi, envisagé de suggérer un processus permettant la réduction de biais fondé sur le travail émotionnel, nécessaire à distance (Svensson, 2014), agrégé au niveau du groupe afin de tenter de limiter les biais de la décision collaborative à distance. Cet outil tend à limiter le conformisme ou le consensus prescrit, en reposant sur la régulation des émotions nécessaires à la prise de décision (Damasio, 1995), à la collaboration de groupe (Sandelands et St Clair, 1993, p. 445) et à l'universalité (Ekman et Oster, 1979). Grâce à l'information, chaque individu prend conscience des biais décisionnels (cognition) auquel sa décision est soumise au sein d'un groupe et régule les émotions qui y sont liées. Ceci confirme non seulement les dires de Kisfalvi (2006) pour qui la subjectivité et les émotions peuvent être des biais si elles sont occultées et devenir une source de connaissances si on se donne la peine de les identifier et de les analyser. Cela corrobore également l'analyse de Stasser et Dietz-Uhler (2001) selon qui les membres du groupe corrigent mutuellement leurs erreurs et mettent en commun des ressources complémentaires. Nos propositions s'articulent également autour de facteurs de contingence de ce travail émotionnel de groupe propre à la décision collaborative à distance. La structure du groupe (design, type de tâches et relations sociales au sein du groupe) et le leader-accompagnateur sont des outils pour manager la réduction de biais de ce type de décision (figure 1).

Le processus « Rapide et Lent» ou «Penser et Ressentir» RALENPTIR, de stratégie de réduction de biais de la décision collaborative à distance, issu de notre analyse est dessiné en figure 1.

Contrairement à Babcock et al. (1997) qui proposent de créer une convergence dans les échanges en évitant les «contradicteurs', notre modèle ralenptir favorise la pensée dissonante. A nouveau, les écrits des dirigeants de Google (Schmidt et Rosenberg, 2014) appuient notre vision. Comme indiqué dans l'introduction, ces auteurs expliquent le consensus par son étymologie : Ressentir ou Penser «To think or feel» (p.2204). Ceci laisse transparaitre l'équilibration dynamique cognition/ émotion. Nous considérons, selon notre analyse, que le consensus s'apparente à ressentir ET penser. Le but : ne pas se soumettre sans conscience et savoir montrer tout désaccord "To surface» (terme cité par les auteurs eux-mêmes p. 2231). Ces auteurs précisent à ce sujet qu'il est important de «travailler en surface» à une étape précise du processus de décision. "To surface» doit intervenir, selon eux, lors des premières réflexions. Or, le travail en surface précède justement le travail en profondeur selon Hochschild (1979). Ainsi, le fait que certains individus fassent l'effort de montrer leur désaccord et leurs dissonances ressentis dès l'entrée du processus décisionnel, limiterait, par une remise en question, les biais collectifs du groupe et favoriserait une analyse en profondeur ultérieure allégée de ces biais. De plus, pendant la décision, puisque le deep-acting n'est pas immédiat, les moments de silence ou de recul, concevables à distance grâce à une temporalité plus modérée, pourraient s'avérer nécessaires à une introspection.

Etant donnée l'interprétation émotionnelle que peut provoquer la relation médiée par ordinateur, la stratégie de réduction des biais par une auto-régulation émotionnelle est d'autant plus importante et réalisable lors de prises de décisions collaboratives à distance, à condition qu'elle devienne un système de prise de décision, établi comme un fonctionnement récurrent.

Pour conclure, cet écrit comprend des limites, liées notamment au manque d'écrits sur les biais de la décision collaborative à distance qui a conduit à synthétiser une littérature

14. Lydia Nicollet (1998), La prise de décision collective : un processus démocratique? Quelques éléments de méthode pour faciliter les prises de décision collectives, Dialogues, propositions, histoires pour une citoyenneté mondiale, $\mathrm{n}^{\circ} 12$.

15. https://atelier.bnpparibas/life-work/article/interface-naturelle-rend-travail-collaboratif-humain 
extrêmement abondante sur la prise de décision et les biais décisionnels individuels et collectifs. Ces limites ouvrent à de nombreuses recherches futures qui auront à tester les différentes propositions décrites sur le concept de travail émotionnel de groupe en lien avec la décision collaborative à distance dans une vision orientée individus. Outre la revue de littérature qui synthétise les études antérieures, l'apport académique de cette réflexion est de pointer le besoin d'équilibration émotion/cognition et de travail émotionnel "de groupe», en tant qu'outil de réduction des biais de la décision collaborative à distance. Ce concept est apparu comme «logique» après avoir réalisé une revue de littérature au travers de domaines de recherche transversaux, depuis la psychologie, sociologie jusqu'à la gestion, en passant par l'économie expérimentale et la neurologie. Chaque domaine montre que l'émotion correspond à un aspect essentiel des biais décisionnels, surtout, collectifs et collaboratifs à distance. L'apport managérial se situe, ainsi, au niveau d'un management de la décision médiée, vouée à se développer à l'avenir. Notre réflexion détaille, en effet, les facteurs et le cheminement espéré par certaines entreprises pour obtenir des collaborateurs une décision médiée commune, non uniforme. Elle présuppose une recherche d'équilibre entre émotion et cognition qui demande de fournir le capital temporel et cognitif aux décideurs pour limiter les biais et réguler les émotions. Au travers de modèles multi-agents orientés-individus, les recherches futures auront, ainsi, à développer l'aspect émotionnel dans l'étude des «debiasing» collaboratifs à distance et les nombreux facteurs de contingence internes et externes.

\section{Bibliographie}

AвrAнам Rebecca (1998). «Emotional Dissonance in Organizations: a conceptualization of consequences, mediators and moderators ", Leadership et Organization Development Journal, vol. 19, $\mathrm{n}^{\circ} 3$, p. 137-146.

Allison Graham (1971). Essence of decision: Explaining the Cuban missile crisis, http://davidmorey.org/rm020pptessense.pdf
ANDERSON, Terry (2004). «Teaching in an online learning context», dans Theory and practice of online learning, édité par Terry Anderson, AU Press, Athabasca University, lère edition, p. 273-294.

Arkes Hal R.; Catherine Blumer (1985). «The Psychology of Sunk Cost», Organizational Behavior and Human Decision Processes, vol. 35, p. 124-140.

Arrow Kenneth J. (1951). Choix collectifs et préférences individuelles, [1998], Paris, Diderot Eds, 234 p.

BABCock, Linda; Loewenstein, George; IssaCharof, Samuel (1997). "Creating Convergence : Debiasing Biased Litigants", Law and Social Inquiry, vol. 22, p. 913-925.

BALAJI Sathyaprakash M.; Chakrabarti, Diganta (2010). «Student Interactions in Online Discussion Forum : Empirical Research from «Media Richness Theory» Perspective», Journal of Interactive Online Learning, vol. 9, $\mathrm{n}^{\circ}$ 1, p. 1-22.

Baltes Boris B., Dickson, Marcus W.; Sherman, Michael P.; Bauer Cara C.; LaGanke, Jacqueline S. (2002). «ComputerMediated Communication and Group Decision Making : A Meta-Analysis", Organizational Behavior and Human Decision Processes, vol. 87, n 1 , p. 156-179.

Boot, Arnoud W.A.; AnjAN V., Thakor; Tood T., Milbourn (2005). "Sunflower Management and Capital Budgeting», The Journal of Business, vol. 78, $\mathrm{n}^{\circ} 2$, p. 501-527.

Boudon Raymond (2002). «La théorie du choix rationnel contre les sciences sociales?» Sociologie et sociétés, vol. 34, n 1, p. 9-34.

Bornstein Gary, Kugler, Tamar; Ziegelmeyer, Anthony (2004). "Individual and group decisions in the centipede game : Are groups more "rational" players?", Journal of Experimental Social Psychology, vol. 40, p. 599-605.

Brunsson, Nils (1982). «The irrationality of action and action rationality: decisions, ideologies and organizational actions", Journal of Management Studies, vol. 19, $\mathrm{n}^{\circ}$ 1, p. 29-44.

Connolly, Terry; Jessup Leonard M.; VAlacich, Joseph S. (1990). "Effects of anonymity and evaluative tone on idea generation in computer-mediated groups", Management Science, vol. 366, p. 689-703.

FIGURE 1

Processus en recherche d'équilibre Emotion/Cognition pour prendre la décision collective à distance : RALENPTIR

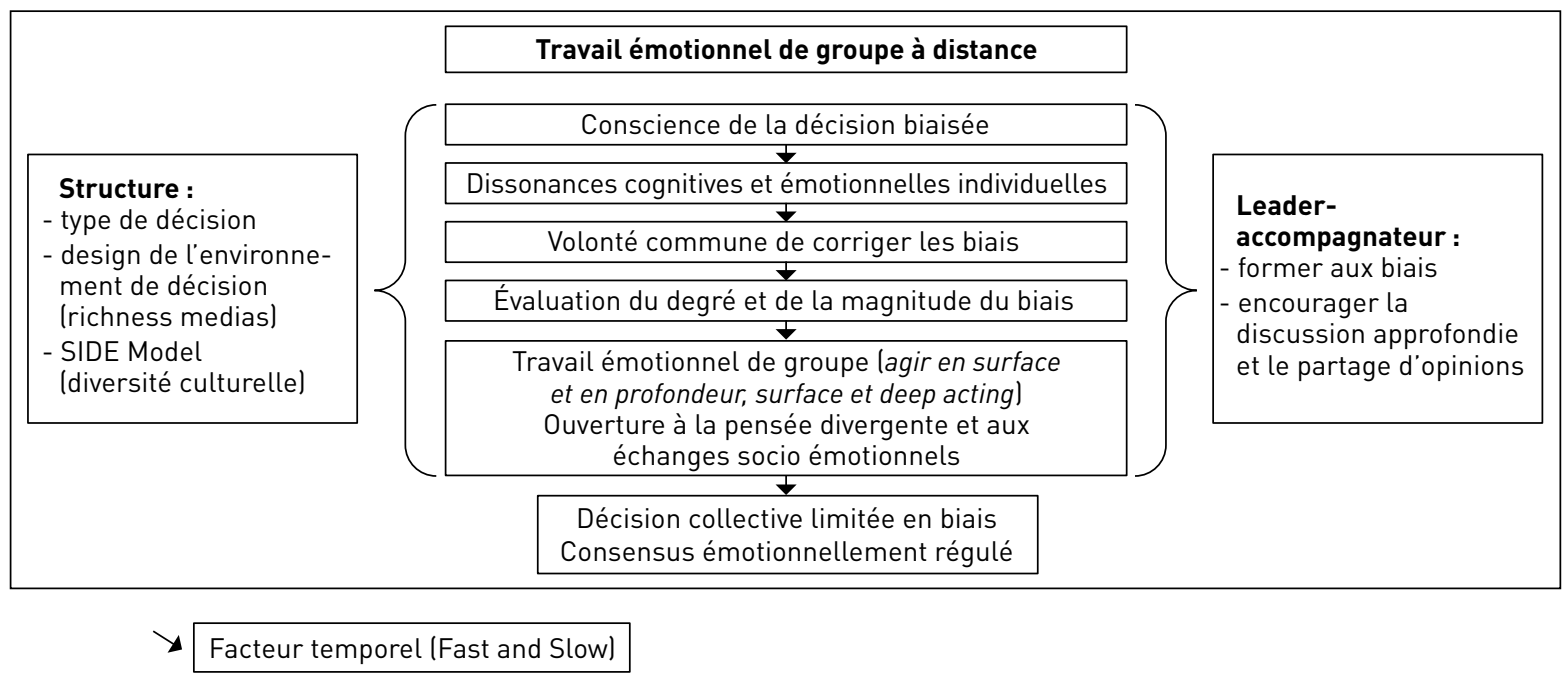


Cомтет, Isabelle (2006). «L'usage du groupware ou la construction d'un dispositif sociotechnique». Revue française de gestion, vol. $168-169$, n $^{\circ}$, p. 287-301.

DAFT, Richard L.; Lengel Robert H. (1986). «Organizational information requirements, media richness and structural design», Management science, vol. 32, $\mathrm{n}^{\circ}$ 5, p. 554-571.

Damasio Antonio, R. (2003). Spinoza avait raison: Joie et tristesse, le cerveau des émotions, Paris, Odile Jacob, $346 \mathrm{p}$.

Damasio, Antonio R. (2000). «A second chance for emotion », In Richard D. R. Lane, L. Nadel, G. L., Ahern, J. Allen et alfred W. Kaszniak (eds.), Cognitive Neuroscience of Emotion. Oxford University Press, p. 12-23.

Damasio, Antonio, R. (1995). L'Erreur de Descartes : la raison des émotions, Paris, Odile Jacob, 368 p.

DARLING-Hammond, Linda, Richardson Nikole (2009). « Research review/teacher learning: What matters.», Educational leadership, vol. 66. $\mathrm{n}^{\circ} 5$, p. 46-53.

Deci, Edward L.; Ryan, Richard M. (1985). Intrinsic Motivation and Self-determination in Human Behavior. New York: Plenum.

De Martino, Benedetto; Kumaran, Dharshan; Seymour, Ben; DolAN, Raymond J. (2006). «Frames, Biases, and Rational Decision-Making» in the Human Brain, Science, vol. 313, n 5787, p. 684-687.

Derks Daantje; Fischer Agneta H.; Bos Arjan E.R. (2007). «The role of emotion in computer-mediated communication : A review», Computers in Human Behavior, vol. 23, p. 842-849.

DeSanctis, Gerardine; Gallupe, R. Brent (1987). «A Foundation for the Study of Group Decision Support Systems », Management Science, vol. 33, n 5, p. 589-609

Dubrovsky Vitaly J. (1985). «Real-time computer conferencing versus electronic mail», dans Proceedings of the human factors society, vol. 29, p. 380-384, Santa Monica, CA : Human Factors Society.

Dubrovsky Vitaly J.; Kiesler Sara; Beheru; N. Sethna (2009). «The equalization Phenomenon: Status Effects in ComputerMediated and face to Face Decision-Making Groups » HumanComputer Interaction, vol. 6, n², p. 119-146

Ekman Paul; Oster, Harriet (1979). «Facial Expressions of Emotion", Annual Review of psychology, vol. 30, p. 527-554.

El-Shinnawy, Maha; Vinze, Ajay. S. (1997). «Technology, culture and persuasiveness : A study of choice-shifts in group settings", International Journal of Human-Computer Studies, vol. 47, p. $473-496$

Festinger, Leon (1957). A Theory of cognitive dissonance. Stanford, CA : Stanford University Press. 291 p.

FIsCHOFF, Baruch (1975). «Hindsight is not equal to foresight : The effect of outcome knowledge on judgment under uncertainty» Journal of Experimental Psychology: Human Perception and Performance, vol. 1, n 3, p. 288-299.

Garrison, D. Randy; Cleveland-Innes Martha (2005). «Facilitating Cognitive Presence in Online Learning: Interaction Is Not Enough ", The American Journal of Distance Education, vol. 19, n' 3 , p. 133-148

Gautier, Anaïs (2015). «L'erreur dans la prise de décision en situation, Le cas d'une collision aviaire en phase d'écopage», Revue Française de Gestion, vol. 41, n² 246, p. 41-62.
GigerenZer, Gerd; David J. Murray (1987). Cognition as intuitive statistics. Hillsdale, NJ, $214 \mathrm{p}$.

Grandey, Alicia A. (2000). «Emotion regulation in workplace : A new way to conceptualize emotional labor. "Journal of Occupational Health Psychology, vol. 5, nº 1, p. 95-100.

Grandey, Alicia A. (2003). «When "the show must go on" : Surface acting and deep acting as determinants of emotional exhaustion and peer-rated service delivery." Academy of Management Journal, vol. 46, p. 86-96.

GRAY, Jeremy R. (1999) «A bias toward short-term thinking in threat-related negative emotional state ", Personality and Social Psychology Bulletin, vol. 25, p. 65-75.

Grimm, Volker; Steven F. Railsback, (2005). Individual-based Modeling and Ecology. Princeton University Press. 485 p.

Hatfield, Elaine; Cacioppo, John T.; Rapson, Ralph L. (1994). Emotional Contagion, Paris : Cambridge University Press, 240 p.

Helmersen Peter; Jalalian Alin., Moran G., Norman F., (2001). Impacts of Information Overload, Eurescom. https://www. eurescom.eu/home.html

Helmreich, Robert L.; Merritt, Ashleigh C. (1998). Culture at work: National, organizational, and professional influences. Aldershot, United Kingdom: Ashgate.

Herring, Susan C. (1996). Computer-mediated Communication: Linguistic, Social, and Cross-cultural perspectives, publié par Susan C. Herring, Editor John Benjamins publishing company, Amsterdam/Philadelphia, $327 \mathrm{p}$.

Hochschild, Arlie R. (1979). «Emotion work, feeling rules, and social structure », American Journal of Sociology, vol. 85, p. 551-575.

Hogarth, Robin M.;. Einhorn Hillel J. (1992). «Order effects in belief updating: The belief-adjustment model», Cognitive Psychology, vol 24, n 1, p. 1-55.

IsEN Alice M. (1984). «The influence of positive affect on decision making and cognitive organization" Advances in Consumer Research, vol. 11, p. 534-537.

JANIs, Irving L. (1982). Victims of groupthink, Boston : Houghton Mifflin

Jessup, Leonard M., Connolly, Terry; Galegher, Jolene (1990). "The effects of anonymity on GDSS group process in automated group problem-solving», MIS Quarterly, vol. 143, p. 312-321.

Kahneman, Daniel (2011). Thinking, fast and slow. New York : Publisher : Farrar, Straus and Giroux, 499 p.

Kahneman, Daniel (2003). "A perspective on judgment and choice : Mapping bounded rationality» American Psychologist, 58, vol. 9, p. 697-720.

Kahneman, Daniel; Tversky, Amos (1984). «Choices, values, and frames». American Psychologist, vol. 39, n 4, p. 341-350.

Keen, Peter G. W.; Scott Morton, Michael S. (1978). Decision Support Systems : An Organizational Perspective, AddisonWesley, Reading, Massachusetts.

Kennedy, Jane (1993). «Debiasing Audit Judgment with Accountability : A Framework and Experimental Results », Journal of Accounting Research, vol. 31, n², p. 231-245.

Kisfalvi, Veronika (2006). «Subjectivity and Emotions as Sources of Insight in an Ethnographic Case Study : A Tale of the Field », M@n@gement, vol. 9, n³, p. 117-135. 
KLeIN, Gary. (1998), Sources of Power How People Make Decisions, MIT Press, $352 \mathrm{p}$.

Kocher, Martin G.; Sutter, Matthias (2007). «Individual versus group behavior and the role of the decision making procedure in gift-exchange experiments ", Empirica, vol. 34, n ${ }^{\circ}$, p. 63-88.

Kocher Martin, Strauß, Sabine; Sutter, Matthias (2004). «Individual or team decision-making - Causes and consequences of selfselection", Economics Letters, vol. 81, n³, p. 341-347.

Kugler, Tamar, Kausel, Edgar E.; Kocher, Martin. G. (2012). "Are groups more rational than individuals? A review of interactive decision making in groups». Wiley Interdisciplinary Reviews : Cognitive Science, vol. 3, n 4, p. 471-482.

Lea, Martin; Spears, Russel (1991). "Computer-mediated communication, de-individuation and group decision-making", International Journal of Man-Machine Studies, (Special issue on «Computer supported cooperative work and groupware»), vol. 39, p. 283-301.

LeA, Martin; Spears, Russel (1992). «Paralanguage and social perception in computer-mediated communication ", Journal of organizational computing, vol. 2, p. 321-341.

LABORIE, Fabrice (2006). «Le concept de salle de décision collective et son application aux processus complexes EADS», Thèse de doctorat, Université Paul Sabatier, Toulouse (France)

LARRICK, Richard (2004). «Debiasing», In the Blackwell Handbook of Judgment and Decision Making, Derek J. Koehler (Editor), Nigel, Harvey (Editor), 684 p.

Lebraty Jean-Fabrice; Pastorelli-Nègre Ivan (2004). «Biais cognitifs : quel statut dans la prise de décision assistée?», Systèmes d'Information et Management, vol. 9, n³, p. 87-115.

LEDoux Joseph E. (2000). «Emotion Circuits in the Brain», Annual Review of Neuroscience, vol. 23, p. 155-184.

LeDoux Joseph E. (1993). «Emotional memory systems in the brain», Behavioral Brain Research vol. 58, p. 69-79.

LefLeY, Frank (2006). «Can a project champion bias project selection and, if so, how can we avoid it?» Management Research News, vol. 29, nº 4, p. 174-183.

Lerner, Jennifer S., Li, Ye; Valdesolo, Piercarlo; Kassam Karim S. (2015). «Emotion and Decision Making», Annual Review of Psychology, vol. 66, n³3, p. 799-823

LEwIN Kurt (1952). «Group decision and social change», dans G. Swanson, T. Newcomb \& E. Hartley (eds)., Readings in social psychology. New York : Henry Holt, p. 459-473.

Mansor Zuraina D., Mazlina Mustaffa, Lailawati M. Salleh (2015). «Motivation and Willingness to Participate in Knowledge Sharing Activities Among Academics in a Public University", Procedia Economics and Finance, vol. 31, p. 286-293.

MCGRATH Joseph E. (1984). Groups : interaction and performance, University of Illinois, Urbana

Prentice-Hall, Inc., Englewood Cliffs, N.J., 165 p.

Marakas, George M. (1999). Decision Support Systems in the Twenty-First Century, Prentice Hall, Upper Saddle River, (N.J., USA), $528 \mathrm{p}$.

Masclet, David; Colombier, Nathalie; Denant-Boemont, Laurent; LoHÉAC, Youenn (2009). «Group and individual risk preferences : A lottery-choice experiment with self-employed and salaried workers. » Journal of Economic Behavior and Organization, vol. 70, $\mathrm{n}^{\circ} 3$, p. 470-484.
Mintzberg, Henry; Waters, Jim (1990). «Studying Deciding: An Exchange of Views Between Mintzberg and Waters, Pettigrew, and Butler», Organizational Studies, vol. 11, n 1, p. 1-6.

Morel, C. (2002). Les décisions absurdes. Sociologie des erreurs radicales et persistantes. Paris: Gallimard.

Milch Kerry F., Weber Elke U.; Appelt Kirstin C.; Handgraaf Michel J.J.; Krantz David H. (2009) «From individual preference construction to group decisions : Framing effects and group processes", Organizational Behavior and Human Decision Processes, vol. 108, p. 242-255.

Mione Anne; Leroy Maya (2013). «Décisions stratégiques dans la rivalité entre standards de qualité : le cas de la certification forestière» Management international / International Management / Gestión Internacional, vol. 17, n² 2, p. 84-104

Neale, Margaret A., Bazerman Max H.; Northcraft Gregory B.; Alperson Carol (1986). "“Choice shift” effects in group decision : A decision bias perspective» International Journal of Small Group Research, vol. 23, p. 33-42.

Nisbett, Richard; DeCamp Wilson Timothy (1977). «The halo effect : evidence for unconscious alteration of judgements", Journal of personality and social psychology, vol. $35, \mathrm{n}^{\circ} 4$, p. 250-256.

Novak Stéphanie; Ulfarino Philippe. (2017). «Nouvelles approches de la décision collective : une introduction», Négociations, vol. 1, $\mathrm{n}^{\circ} 27$, p. $67-71$.

O'GRADY Laura; JADAD Alejandro R (2008). «Shifting from shared to collaborative decision making : a change in thinking and doing». Journal of Participatory Medicine, vol.2, $\mathrm{n}^{\circ} 13$, p.1-6.

O’Reilly, Charles. A.; Roberts, Karlene. H. (1977). «Task group structure, communication, and effectiveness in three organizations ». Journal of Applied Psychology, vol. 62, n 6, p. 674-681.

Paese, Paul W.; Bieser, Mary; Tubbs, Mark E (1993). «Framing effects and choice shifts in group decision making», Organizational Behavior and Human Decision Processes, vol. 56, p. 149-165.

Pastorelli, Ivan (2000). «L'impact d'un outil de contrôle sur l'organisation : le cas de l'atelier industriel de l'aéronautique», Université de Nice Sophia-Antipolis - Rodige.

Pontikes, Patricia; Barnett William (2017). «The Non-consensus Entrepreneur : Organizational Responses to Vital Events», Administrative Science Quarterly, vol. 62, $\mathrm{n}^{\circ}$ 1, p. 140-178.

RaI, Arun; StubBart, Charles; PAPER, David. (1994). «Can Executive Information Systems Reinforce Biases?», Banking Technology, vol. $4, \mathrm{n}^{\circ} 2$, pp. 87-106.

Raghunathan, Rajagopal; Pham Michel T. (1999). «All negative moods are not equal : Motivational influences of anxiety and sadness on decision making», Organizational Behavior and Human Decision Processes, vol. 79, p. 56-77.

Reason, James (2013) L'erreur humaine, Paris, Presses des Mines, collection Economie-Gestion, (traduit par J.M. Hoc, de Human error, 1990, Cambridge University Press), 210 p.

Reid Fraser J. M., Linden J. Ball, Andrew M. Morley, Jonathan St B. T. Evans (1997) «Styles of group discussion in computermediated decision making», Social Psychology, vol. 36, n³, p. 241-262.

REID, Elizabeth M. (1991). Electropolis : Communication and community on Internet relay Chat, Unpublished thesis, Department of History, University of Melbourne, available http://www.irchelp.org/communication-research/academic/ academic-reid-e-electropolis-1991.html 
RICE, Ronald E. (1984). «Mediated group communication», dans the new media: Communication, research and technology, R.E. Rice et associates (Eds.), p. 129-156. Beverly Hills, CA : Sage.

Roy, Bernard; Bouyssou Denis (1993). «Aide multicritère à la décision : méthodes et cas». Economica, Paris, 695 p.

Roy, Marie-Christine; F.; LERCH Javier (1996) «Overcoming Ineffective Representations in Base-Rate Problems ", Information Systems Research, vol. 7, n², p. 233-247.

Sandelands, Lloyd E.; St. Clair Linda. (1993). «Toward an empirical concept of group", Journal for the Theory of Social Behavior, vol. 23, p. 423-458.

Schmidt, Erik; Rosenberg Jonathan (2014). How Google works, Great Britain, John Murray (Publishers) 352 p.

Schwarz, Norbert; Clore Gérald L. (2003). «Mood as information : 20 years later», Psychological Inquiry, vol. 14, p. 296-303.

Schwenk, Charles R. (1989) «linking cognitive, organizational and political factors in explaining strategic change», Journal of Management Studies, vol. 26, n² 2, p. 177-187.

Sechrist, Gretchen B.; Charles, Stangor (2001) «Perceived consensus influences intergroup behavior and stereotype accessibility» Journal of Personality and Social Psychology, vol 80, nº 4 , p. 645-654.

Siegel, Jane; Dubrovsky, Vitaly; Kiesler, Sara; McGuire, TimothyW (1986). «Group processes in computer-mediated communication». Organizational Behavior and Human Decision Processes, vol. 37, p. 157-187.

Simon, Herbert (1945) Administration et processus de décision, Economica, Paris, (trad. 1983), 322 p.

Skitka, Linda J.; Mosier, Kathleen L.; Burdick, Mark. (1999). «Does automation bias decision-making», International Journal of Human-Computer Studies, vol. 51, n 5, p. 991-1006.

SKyrik, Petr. (2010). «Proposal of a model for effective management and development of virtual teams ", Journal of Systems Integration; vol. $1, \mathrm{n}^{\circ} 4$, p. 27-38.

Smoliar, Stephen W.; Sprague Ralph (2002). «Communication and Understanding for Decision Support», Proceedings of International Conference IFIP TC8/WG8.3, p. 107-119, Cork (Ireland).

Soll, Jack B., Milkman Katherine L.; Payne John W. (2013). A User's'Guide to Debiasing. www.opim.wharton.upenn. edu/ kmilkman/Soll_et_al_2013.pdf

Song, Fei (2008). «Trust and reciprocity behavior and behavioral forecasts : Individuals versus group-representatives», Games and Economic Behavior, vol. 62, n 2, p. 675-696

Stasser, Garold; Dietz-Uhler, Beth. (Eds.) (2001). Collective Choice, Judgement and

Problem Solving, Blackwell handbook of social psychology: Group processes.Wiley-Blackwell, $712 \mathrm{p}$.
STASSER, Garold; Stewart, Dennis (1992). «Discovery of hidden profiles by decision-making groups : Solving a problem versus making a judgment", Journal of Personality and Social Psychology, vol. $63, \mathrm{n}^{\circ} 3$, p. $426-434$.

Stasser, Garold, Taylor, Laurie. A., Hanna Coleen (1989). «Information sampling in structured and unstructured discussions of three and six-person groups ", Journal of Personality and Social Psychology, vol. 57, $\mathrm{n}^{\circ}$ 1, p. 67-78.

Stasser, Garold; Titus William (1985). «Pooling of unshared information in group decision making: Biased information sampling during discussion.» Journal of Personality and Social Psychology, vol. 48, nº, p. 1467-1478.

Strauss, Susan G., (1996). «Getting a clue : the effects of communication media and information distribution on participation and performance in computer-mediated and face-to-face groups", Small group research, vol. 27, n 1, p. 115-142.

Svensson, Jakob (2014) «Power, Identity, and Feelings in Digital Late Modernity, The Rationality of Reflexive Emotion Displays Online», dans Internet and Emotions, Edited by Tova Benski and Eran Fisher, New-York, p. 17-32.

Turban, Efraim; Jay E. Aronson, Ting-Peng, Liang (2001). Decision support systems and intelligent systems, $6^{\text {th }}$ Prentice-Hall International, Upper Saddle River (N. J., USA), 930 p.

TURKLE S., (1995). Life on the screen: Identity in the age of the Internet, New York, Simon \& Schuster, 352 p.

Tversky, Amos; Kahneman, Daniel (1974). «Judgment under Uncertainty : Heuristics and Biases ", Science, New Series, vol. $185, \mathrm{n}^{\circ} 4157$, p. $1124-1131$

VAn Hoorebeke, Delphine (2008). «l'émotion et la prise de décision », Revue française de gestion, vol. 2, n 182, pp. 33-44.

WALTHER, Joseph B. (1996). «computer-mediated communication : impersonal, interpersonal, and hyperpersonal interaction", Communication Research, vol. 23, nº 3, p. 3-43.

Volery, Thierry; Lord Deborah. (2000). «Critical success factors in online education. " International journal of educational management, vol. 14. $\mathrm{n}^{\circ}$ 5, p. 216-223.

Whyte, Glen (1993). «Escalating commitment in Individual and Group Decision Making : A prospect Theory Approach", Organizational Behavior and Human Decision Processes, vol. 54, p. $430-455$.

Wilson, Timothy D., David B. Centerbar; Nancy, Brekke (2002). "Mental contamination and the debiaising problem", dans Heuristics and Biases: The psychology of Intuitive Judgment, edited by Thomas Gilovich, Dale Griffin et Daniel Kahneman, Cambridge University Press, USA, 882 p.

YANIV, Ilan (2011) "Group diversity and decision quality : Amplification and attenuation of the framing effect ", International Journal of Forecasting, vol. 27, p. 41-49.

Zigurs, Ilze, Scott Poole M.; DeSAnCtis Gerardine L. (1988). «A Study of Influence in Computer-Mediated Group Decision Making» MIS Quarterly, vol. 12, n 4, p. 625-644. 